\title{
SISTEMA DE VISIÓN PARA EL GUIADO DE UN ASISTENTE ROBÓTICO EN OPERACIONES DE CIRUGÍA ENDONASAL
}

\author{
Ander Loidi Yarza, Rafael Estop Remacha, Eusebio de la Fuente, Juan Carlos Fraile, J. Pérez Turiel \\ ITAP Univ. Valladolid. Paseo Cauce, 59. 47011 Valladolid, \\ email: ander.loidi@alumnos.uva.es, rafael.estop@alumnos.uva.es, \\ efuente@eii.uva.es, jcfraile@eii.uva.es, turiel@eii.uva.es
}

\begin{abstract}
Resumen
La cirugía endoscópica endonasal transesfenoidal es una técnica mínimamente invasiva que presenta numerosas ventajas al permitir a los cirujanos un acceso rápido y directo a determinados tumores cerebrales a través de las fosas nasales. Sin embargo, este tipo de cirugía requiere de la participación de dos cirujanos que deben operar con muchas restricciones de espacio. En este artículo se presenta un sistema de guiado para un robot quirúrgico basado en la información proporcionada por una cámara ToF (time of flight) y la información preoperatoria obtenida mediante una serie de imágenes de resonancia magnética. Este sistema formará parte de un asistente robótico que gestione el manejo del endoscopio de forma autónoma.
\end{abstract}

Palabras clave: robótica quirúrgica; cirugía endonasal; imágenes de resonancia magnética MRI; PCL Point Cloud Library.

\section{INTRODUCCIÓN}

La cirugía de la base del cráneo es un campo que ha experimentado un fuerte desarrollo en los últimos años. La necesidad de acceder a la región selar del cráneo, ubicada en la zona inferior de la envoltura craneal, es relativamente frecuente en la práctica neuroquirúrgica. En esta zona pueden sucederse diferentes afecciones que necesiten de una intervención como por ejemplo los adenomas hipofisarios, más conocidos comúnmente como tumores [12][13]. La cirugía endoscópica endonasal transesfenoidal simplifica el acceso a la región selar evitando la disección y posterior reconstrucción de estructuras nasales o septales. De esta forma se reducen los inconvenientes funcionales y estéticos derivados de las otras técnicas, así como una reducción de los tiempos de recuperación tras la operación [9][6].

Por otro lado, los continuos avances técnicos en sistemas de navegación, técnicas de adquisición y tratamiento de imágenes y robótica están acelerando la evolución de la cirugía de la base del cráneo.

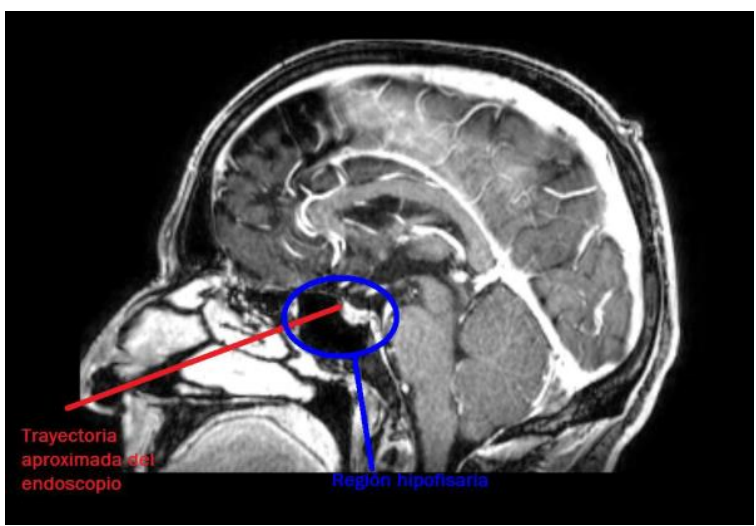

Figura 1. Trayectoria del endoscopio (en rojo) en una intervención endonasal para acceder a región hipofisaria (en azul).

Existen estudios realizados sobre cadáveres que demuestran la viabilidad y las ventajas de la cirugía robótica de la base del cráneo [8][5]. Actualmente, el procedimiento que llevan a cabo los cirujanos no es ergonómico y es técnicamente difícil por consideraciones de espacio. La dificultad radica no solo en la ubicación de los instrumentos intranasalmente, sino también en la colocación de las cuatro manos en un área relativamente pequeña fuera de la nariz ya que el cirujano introduce dos herramientas por la fosa nasal derecha, mientras que el asistente maneja el endoscopio y un aspirador quirúrgico por la fosa nasal izquierda [4]. En este artículo se propone un sistema de guiado para un asistente robótico que gestione el manejo del endoscopio de forma autónoma. Este sistema de guiado se basará en la información tridimensional de la cabeza del paciente y de la serie de imágenes de resonancia magnética (MRI) captadas en una etapa preoperatoria.

A continuación, se presenta la estructura del artículo. En la siguiente sección se expone el sistema para la adquisición y preprocesamiento de las imágenes 3D de la cabeza del paciente. En este apartado se justifica además la necesidad de varias imágenes para la reconstrucción 3D. El tratamiento realizado sobre las imágenes MRI para generar la nube tridimensional de puntos con esta información se presenta en el apartado siguiente. En la sección 4 se detalla el procesamiento realizado para obtener una representación $3 \mathrm{D}$ de las 
fosas nasales del paciente. A continuación, se registra esta información con la posición real de la cabeza del paciente en la mesa de operaciones con objeto de establecer la trayectoria del asistente robótico. Finalmente se presentan las conclusiones de este trabajo.

\section{ADQUISICIÓN $\mathbf{Y}$ PREPROCESAMIENTO DE IMÁGENES 3D DE LA CABEZA DEL PACIENTE}

Para la adquisición de la información tridimensional de la cabeza del paciente se ha utilizado una cámara ToF (ToF - Time offlight) de la marca Basler, modelo ToF640-20gm. La cámara se ha desplazado para la toma de varias imágenes mediante el robot colaborativo de la marca Universal Robots y modelo UR3.

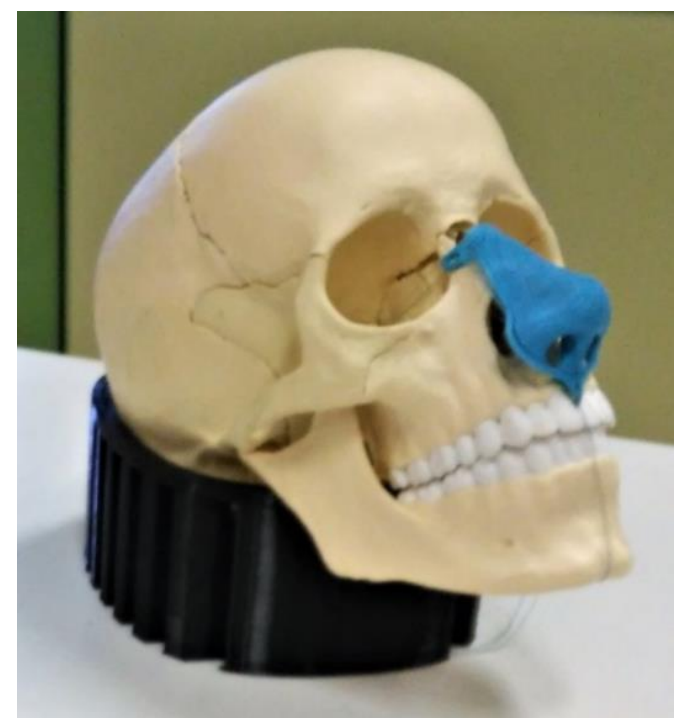

Figura 2. Modelo de cráneo humano al que se le ha incorporado un apéndice nasal de plástico para las pruebas.

La cámara proporciona las coordenadas tridimensionales de los puntos de la escena referidos a un sistema con origen en su centro óptico expresadas en milímetros. Además, para cada punto proporciona un cuarto parámetro correspondiente su nivel de gris.

La cámara tiene una resolución VGA (640x480), por tanto, las imágenes capturadas tienen 307.200 puntos y es conveniente reducir este conjunto tan elevado de datos, eliminando puntos irrelevantes, para facilitar el procesamiento y limitar los tiempos de ejecución. Para, para ello inicialmente se han aplicado dos filtros empleando las librerías $P C L$ (Point Cloud Library)[10]:
- Filtro de distancia: Dado que conocemos el entorno en el cual se va a trabajar, se puede asumir en qué rango de distancia aproximadas se encontrará el objeto de interés, el cráneo del paciente. Con un filtro de distancias se eliminan todos los puntos que no estén dentro de este rango dado de profundidad (coordenada $Z$ ).

- Reducción de densidad: Como se ha comentado, debido a la resolución de la cámara y a que los puntos de la nube estarán muy próximos por la corta distancia que separa la cámara del cráneo, es conveniente disminuir el número de datos. Esta reducción se lleva a cabo preestableciendo una distancia mínima entre dos puntos de la nube y prácticamente no supone una pérdida de información.

Por otro lado, las imágenes captadas por la cámara ToF contienen ruido e información innecesaria que se deberán eliminar en la medida de lo posible para lo cual se han empleado los tratamientos siguientes:

- Eliminación de valores atípicos: Estos valores, también llamados outliers, son puntos que generan en la frontera entre objetos y fondo, sin llegar a ser parte de ninguno de los dos. Estos puntos pueden alterar mucho algunos de los procesos posteriores como la búsqueda de puntos clave $y$ se eliminan empleando un filtro estadístico.

- Eliminación de la superficie de fondo: Las imágenes de la cabeza del paciente siempre se capturan estando éste sobre una mesa de operaciones. Esta superficie lógicamente aparecerá también como fondo en la nube de puntos, pero no presenta ningún interés de cara al procesamiento posterior. Los puntos correspondientes a la mesa pueden ser fácilmente detectados y eliminados haciendo una búsqueda de los puntos en la nube que se ajustan a un plano (figura 3).

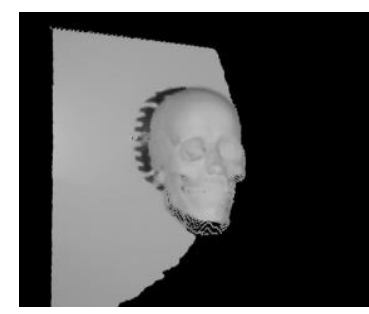

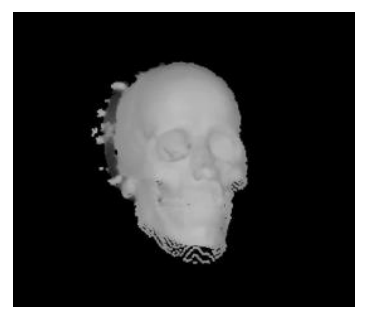

b
Figura 3. Eliminación de la superficie de fondo en las imágenes adquiridas por la cámara ToF. a)Imagen captada. b)Imagen procesada, sin los puntos de fondo. 


\subsection{Adquisición de múltiples imágenes y reconstrucción 3D}

La adquisición de la nube de puntos 3D empleando la cámara $\mathrm{ToF}$ y el preprocesamiento presentado en el apartado anterior proporcionan un buen punto de partida para obtener la información tridimensional que guíe al robot quirúrgico en la maniobra inicial de acercamiento. No obstante, la captura de una única imagen de la cabeza del paciente solo permite adquirir una faceta de ésta, siendo imposible obtener información de la zona oculta a la lente. Por este motivo, y teniendo en cuenta que en nuestra aplicación se precisa reconstruir la cabeza completa del paciente, será preciso tomar varias imágenes desde diferentes posiciones e integrar los datos captados en una sola nube de puntos que defina de la forma más completa posible la escena con la que se va a trabajar después.

El hecho de tomar imágenes desde diferentes puntos de vista plantea el problema de que se deberá conocer perfectamente la posición y la orientación en que se han adquirido cada una de ellas para integrarlas en una sola nube de puntos. Por este motivo se ha empleado un robot colaborativo UR3, que se ha encargado de desplazar la cámara a unos puntos preestablecidos y con orientaciones conocidas.
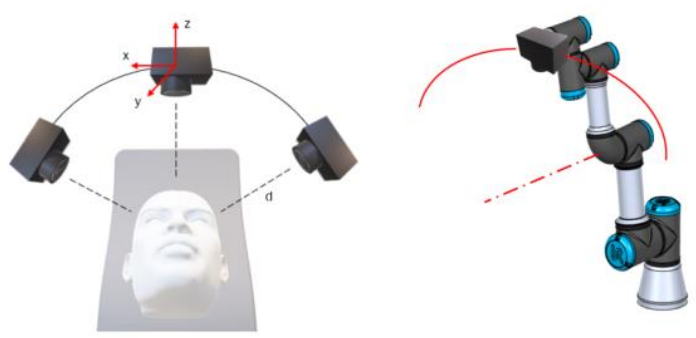

Figura 4. Adquisición de tres imágenes empleando el robot colaborativo para desplazar la cámara ToF

Para realizar correctamente la reconstrucción habrá que tener en cuenta que resulta de gran utilidad configurar el sistema de referencia de la herramienta del robot para que coincida con el de la cámara. De este modo, el programa que mueve el robot podrá determinar la posición y rotación exactos de la cámara en cada momento y por lo tanto se podrán calcular los valores de translación y rotación entre dos puntos cualesquiera de forma sencilla.

Una vez obtenidos los valores de rotación y translación de cada una de las imágenes es posible calcular las diferentes matrices de transformación que consigan que todas las imágenes compartan un mismo sistema de referencia. A partir de este momento, lo único que hay que hacer es integrar las nubes de puntos en una sola para conseguir una reconstrucción completa del objeto.

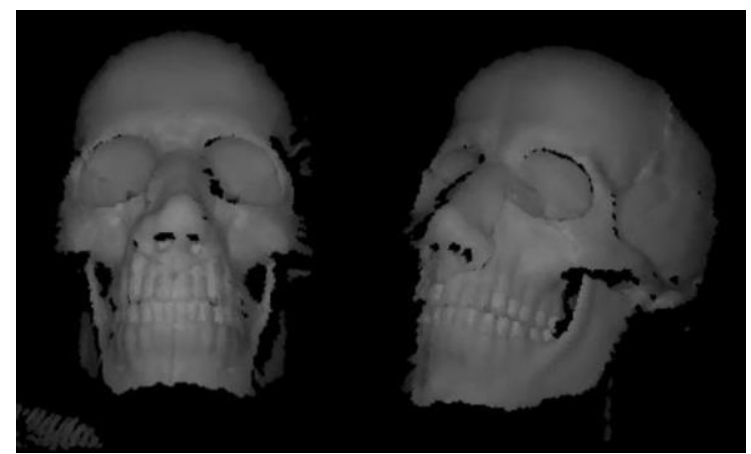

Figura 5. Dos de las tres imágenes captadas por la cámara ToF para la reconstrucción tridimensional del cráneo.

\section{REPRESENTACIÓN 3D DE LAS IMÁGENES MRI}

La información que proporciona la cámara $\mathrm{ToF}$ permite el acercamiento del robot al paciente. No obstante, esta cámara es incapaz de proporcionar información sobre la orientación que deberá tener la herramienta para ser introducida en las fosas nasales con la precisión y garantía que requiere la manipulación de una zona tan delicada. Para esto es preciso conocer la estructura interior del cráneo del paciente.

Esta información del interior de la cabeza del paciente, se obtiene a partir de una resonancia magnética MRI (Magnetic Resonance Imaging) que se debe haber realizado previamente al paciente. La resonancia magnética genera una serie de imágenes axiales del cráneo. El número de imágenes en la serie es variable, dependiendo de la distancia a la que se hagan los cortes axiales.

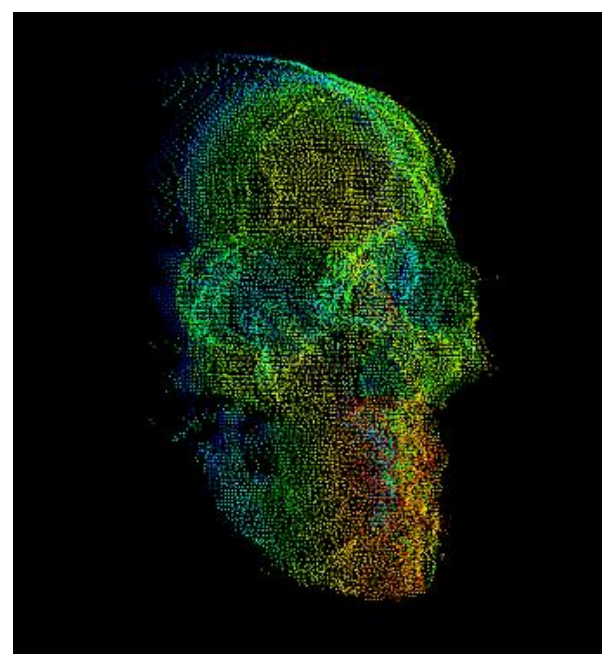

Figura 6. Reconstrucción tridimensional del cráneo a partir de las tres imágenes captadas. 
Lógicamente para poder ubicar espacialmente las estructuras internas que aparecen en las imágenes MRI es preciso alinear estas con el conjunto de datos proporcionados por la cámara ToF. Este proceso, denominado registro de imágenes, consiste en llevar los datos MRI al mismo sistema de coordenadas que emplea la cámara ToF. En el apartado 5 abordaremos con detalle este procesamiento.

Las resonancias magnéticas suelen ser almacenadas utilizando el estándar de imágenes médicas DICOM, sin embargo, este formato no es válido para trabajar con las librerías PCL. Se ha realizado un programa que lee la serie MRI y las reconstruye en una sola nube de puntos. Para conseguir esto hay que tener varios aspectos en cuenta:

- Eliminación del fondo: Cuando se leen imágenes planas correspondientes a una resonancia magnética se puede apreciar que los tejidos y estructuras se encuentran sobre un fondo negro el cual no tiene ninguna información relevante. Este fondo ha de ser eliminado ya que, si no también aparecerá en la nube de puntos, aumentando de forma considerable su tamaño sin aportar ningún beneficio adicional. Para excluir estos datos del fondo basta con imponer una condición sobre la intensidad de luz de tal forma que si un punto es totalmente negro no será copiado la nube de puntos.

- Separación entre imágenes: Será necesario conocer cuántas imágenes han de leerse y la separación que tienen entre ellas para poder así generar una nube con las dimensiones correctas. Esta información está incluida en el fichero DICOM.

- Resolución y tamaño real: Al igual que en el punto anterior, es preciso determinar de antemano qué distancia representa un pixel en la realidad para poder así separar los puntos en concordancia y así obtener una nube que represente fielmente las distancias y proporciones reales. Estos datos también figuran en el fichero DICOM.

- Cantidad de puntos: En nuestro caso contábamos con una serie MRI de 200 imágenes de resoluciones $320 \times 320$ pixeles. Esto significa que si añadimos toda esa información a una única imagen en 3 dimensiones la cantidad de puntos que se tendrá será de aproximadamente 20 millones. Aunque se elimine el fondo, como se ha indicado, siguen siendo demasiados para trabajar de una forma efectiva. Por ello, se recurre nuevamente un filtro que reduce la cantidad de puntos sin alterar a las geometrías ni la información más importante de la imagen.

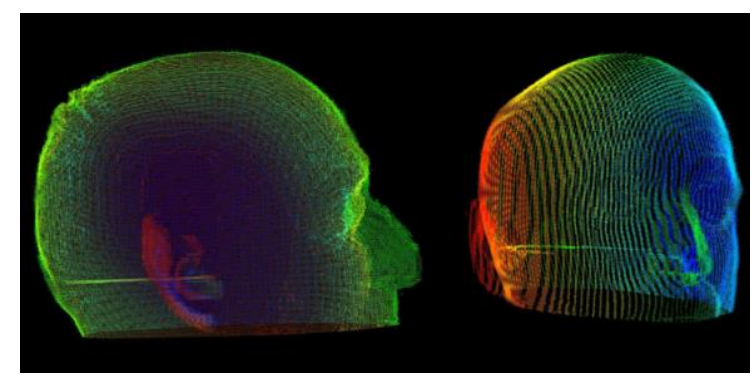

Figura 7. Resultado final de la reconstrucción 3D a partir de la serie MRI. Solo se ha representado el exterior por claridad.

En la figura 7 se muestra el resultado final de la construcción de la resonancia magnética. Resulta importante resaltar que además del contorno, que es lo que se ha representado en la figura por claridad, la nube contiene la información de todo el interior de la cabeza lo que hace que sea muy útil para los procesos posteriores.

\section{EXTRACCIÓN DE LAS FOSAS NASALES EN LAS IMÁGENES MRI}

La forma de la cavidad nasal del paciente se extraerá a partir de los datos contenidos en la serie de imágenes MRI. Para ello se ha determinado una región de interés (ROI) en la cual puede encontrarse la nariz. Para poder especificar las coordenadas de la ROI dentro de la imagen se han tenido en cuenta las medidas y proporciones de una cara humana promediando los datos obtenidos por Leong S.C. y White P.S. [7] quienes establecen que la nariz humana tiene una anchura promedio de 3,75 centímetros y una longitud promedio de 3,425 centímetros.

Para asegurar el encuadre de la nariz en su totalidad dentro de la ROI rectangular, se tomará un valor de 4 centímetros para la anchura. Sin embargo, para la altura de la ROI no bastará con ajustar un poco el valor obtenido ya que las fosas nasales no se apreciarían en su totalidad. Por lo tanto, se determina una altura de 7.5 centímetros para el rectángulo de nuestra ROI.

Una vez establecido el tamaño de la ROI, será preciso ubicar este rectángulo dentro de nuestra imagen. Al encontrarse la cabeza centrada en la serie MRI, es fácil centrar la región de interés verticalmente justo en la mitad de la imagen, es decir, sobre alguno punto del eje representado en amarillo en la figura 8. Para centrarla horizontalmente, se comenzará buscando aquel punto que contenga el mayor valor de la coordenada "Y". Puede que el punto que se encuentre no esté situado sobre el eje amarillo, pero esto no supone ningún problema. En la figura 8 se representa 
una posición aleatoria de dicho índice representado por un punto de color verde. Posteriormente se le resta, al valor "Y" de dicha posición, la mitad del valor de la altura definida para la ROI, y se traza el eje horizontal verde. De esta manera, queda definido el centro, a partir del cual se generará la ROI, como la intersección del eje verde y el eje amarillo. Orientativamente, puede apreciarse la localización del centro de la región en la figura 8 representado por un punto rojo.

Una vez queda definido en qué consiste y cómo se calcula la región de interés, se procede a la obtención de cada uno de los orificios nasales externos, denominados narinas. Para ello se barrerán todas las imágenes del plano axial, y para cada una de ellas se realizarán los siguientes pasos: aplicación de un filtro de suavizado seguido de una binarización adaptativa. A continuación, se procede a ubicar la ROI siguiendo el procedimiento expuesto anteriormente. En la figura 8 se aprecia la ROI sobre la imagen binarizada. La ROI será la única región de la imagen que se procesará de la forma que se expone a continuación.

Sobre la ROI se aplica una operación de cierre morfológico con un elemento estructurador de pequeño tamaño. Esta operación se encargará de cubrir pequeños huecos que puedan surgir en la imagen. Para poder identificar y almacenar únicamente con las narinas en estas secciones se procede a discriminar las distintas regiones en función del valor de su superficie. Es decir, se aplicará un filtro donde se establecen unos límites para los valores de las áreas de manera que solo se almacenen aquellos objetos que cumplan los requisitos. La diferencia entre las áreas en las ROIs de todas las imágenes de la serie es significativa, donde se pueden encontrar elementos con valores muy pequeños (representado por el color verde en la figura 9) o muy grandes (rojo), y valores intermedios (azul), correspondientes a las fosas nasales.
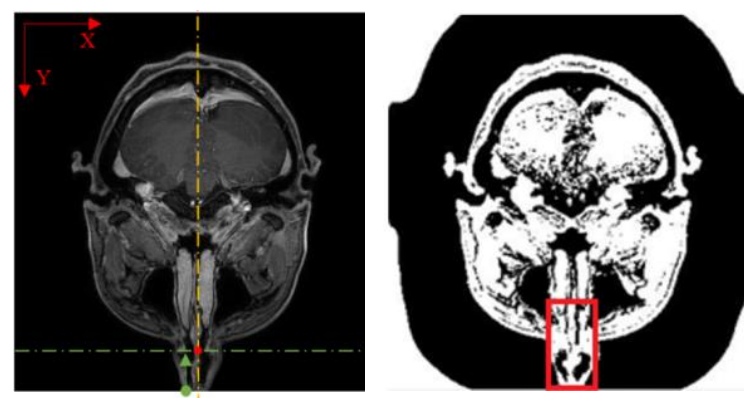

Figura 8: Localización del centro de la región de interés (ROI). ROI delimitada por un rectángulo de bordes rojos sobre la imagen binarizada.
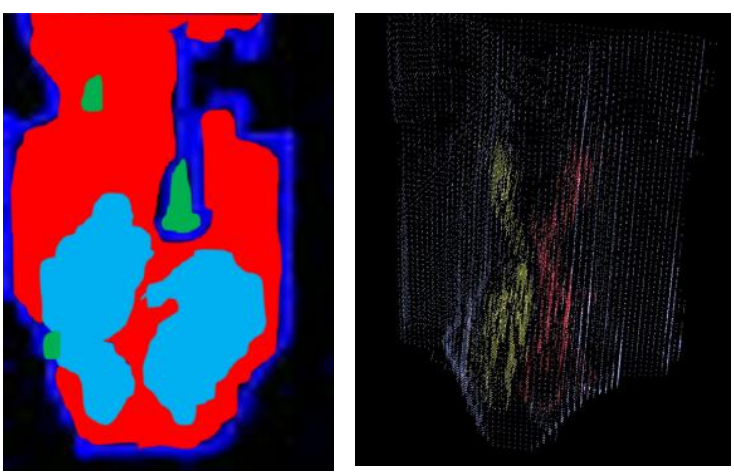

Figura 9. a) Segmentación de las narinas en la ROI. b) Reconstrucción 3D de las fosas nasales.

\section{REGISTRO DE MRI CON IMAGEN TOF}

El proceso desarrollado anteriormente para la reconstrucción 3D de las fosas nasales del paciente no tiene ninguna utilidad si ese resultado no se expresa sobre un sistema de coordenadas ubicado en la mesa de operaciones sobre la que se encuentra el paciente. Es preciso determinar cuál es la transformación que hay que aplicar a la resonancia magnética para que esté alineada con la posición real del paciente. Esta etapa se denomina registro y consiste básicamente en establecer una puesta en correspondencia de las informaciones obtenidas a través de la cámara y través de la resonancia magnética.

Esta fase es posiblemente, la más compleja y crítica del proyecto ya que toda la precisión y limpieza de las nubes de puntos que se ha conseguido en las fases anteriores puede no servir para nada si durante este proceso no se consigue calcular la transformación para que ambas nubes coincidan perfectamente.

Durante el desarrollo del proyecto de investigación se han explorado dos opciones diferentes para el registro. Una primera, totalmente automática, empleando las librerías de PCL, destinadas al reconocimiento de modelos dentro de una escena más compleja. El proceso está definido y explicado en [3] y recurre a diversos algoritmos de obtención de keypoints [2] y de búsqueda empleando la transformada de Hough [11].
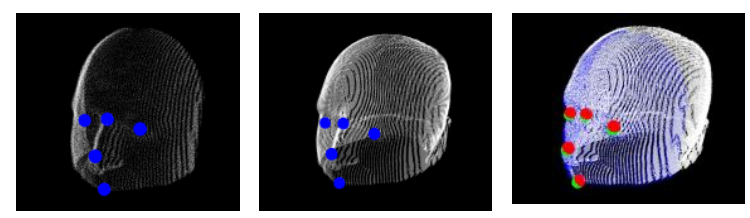

Figura 10. a) Puntos de la cara señalados por el usuario. b) Puntos de la resonancia indicados por el usuario. c) Resultado y desviación del algoritmo ICP. 


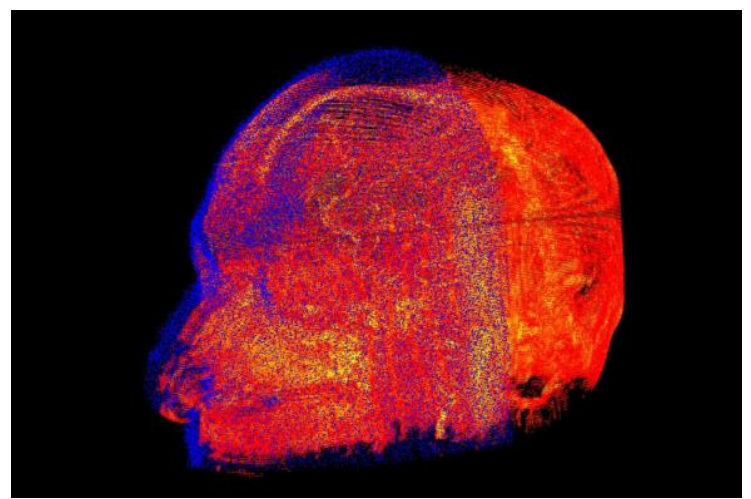

Figura 11. Resultado del algoritmo ICP

Para que este proceso de registro automático funcione correctamente deben establecerse numerosos parámetros que determinarán las tolerancias con las que se admitirán los reconocimientos y la profundidad con la que se debe buscar. El principal inconveniente de este sistema es que dichos parámetros resultan verdaderamente difíciles de ajustar para obtener un funcionamiento robusto con las características que tiene nuestro modelo. Además, este procedimiento lleva consigo una gran carga computacional ya que tanto la escena como el modelo que se utilizan en nuestra aplicación no son simples.

Se ha explorado una segunda técnica para el registrado. Los problemas mencionados en el apartado anterior pueden ser resueltos si solicitamos al usuario que seleccione con el ratón unos puntos clave de forma manual. Estos puntos de referencia pueden ser los ojos, la nariz o la barbilla, ya que dichos puntos son fácilmente reconocibles y la distancia entre ellos no varía en función de la posición o gesto del paciente.

Para ello se utilizará el visualizador de PCL, marcando los puntos seleccionados sobre la imagen capturada por la cámara ToF y el mismo conjunto de puntos sobre la reconstrucción de la resonancia magnética.

Hay que mencionar que no es necesaria una precisión absoluta por parte del usuario para el correcto funcionamiento del método ya que el propio algoritmo conseguirá corregir las posibles desviaciones de los puntos seleccionados.

En el momento en el que se conoce la posición de estos puntos se recurre al algoritmo ICP (Iterative closest point) [3] [1], para que determine la transformación que debe darse al conjunto de puntos modelo para que tenga la posición y orientación de la escena.

Este método de registro resulta ser mucho más fiable y preciso que el anterior ya que además el método ICP proporciona un valor de confianza en los cálculos lo que ayuda a detectar problemas en la estimación, pudiendo volver a solicitar capturas de imagen o de toma de puntos si fuese necesario.

\section{CONCLUSIONES}

El objetivo principal de este trabajo es el desarrollo de una aplicación de visión artificial para el guiado de un robot colaborativo quirúrgico en operaciones de cirugía endoscópica endonasal transesfenoidal.

El sistema de visión presentado es capaz de determinar la posición de la cabeza del paciente durante la intervención quirúrgica a partir de varias imágenes 3D obtenidas por una cámara ToF. Para el guiado del robot, que manejará el endoscopio, resulta necesario referir las estructuras detectadas en la serie MRI al sistema de coordenadas del robot. La reconstrucción tridimensional de fosas nasales ha sido ubicada en el espacio del robot poniendo en correspondencia las imágenes adquiridas por la cámara ToF con las de resonancia magnética realizadas en una etapa preoperatoria.

El sistema de visión presentado está siendo integrado actualmente en el entorno robotizado.

\section{Agradecimientos}

Este trabajo ha sido financiado por el Ministerio de Economía, Industria y Competitividad, a través del proyecto: Sistema Robótico para Neurocirugía Endoscópica Endonasal DPI2016-80391-C3-3-R.

\section{English summary}

\section{VISION SYSTEM FOR THE GUIDANCE OF A ROBOTIC ASSISTANT IN ENDONASAL SURGERY OPERATIONS}

\begin{abstract}
Endoscopic transsphenoidal endonasal surgery is a minimally invasive technique that has numerous advantages by allowing surgeons quick and direct access to certain brain tumors through the nostrils. However, this type of surgery requires the participation of two surgeons who must operate with many space restrictions. This article presents a guidance system for a surgical robot based on the information provided by a ToF (time of flight) camera and the preoperative information obtained through a series of magnetic resonance images. This system will
\end{abstract}


be part of a robotic assistant that manages the handling of the endoscope autonomously.

Keywords: surgical robotics; endonasal surgery; MRI magnetic resonance imaging; PCL Point Cloud Library.

\section{Referencias}

[1] Aldoma, A., Marton, Z., Tombari, F., Wohlkinger, W., Potthast, C., Zeisl, B., ... Vincze, M. (2012). Tutorial: Point Cloud Library: Three-Dimensional Object Recognition and 6 DOF Pose Estimation. IEEE Robotics \& Automation Magazine, 19(3), 8091.

https://doi.org/10.1109/MRA.2012.2206675

[2] Alexandre, L. a. (2012). 3D descriptors for object and category recognition: a comparative evaluation. Workshop on Color-Depth Camera Fusion in Robotics at the IEEE/RSJ International Conference on Intelligent Robots and Systems (IROS), Vilamoura, Portugal, 1(3), 7.

https://doi.org/10.1109/TPAMI.2011.263

[3] Besl, P. J., \& McKay, N. D. (1992). A method for registration of 3-D shapes. IEEE

Transactions on Pattern Analysis and Machine Intelligence, 14(2), 239-256.

https://doi.org/10.1109/34.121791

[4] Dubey SP, Munjal VR. Endoscopic Endonasal Transsphenoidal Hypophysectomy: Two Hand Versus Four Hand Technique: Our Experience. Indian J Otolaryngol Head Neck Surg. 2014 Sep 4;66(3):287-90.

[5] Hanna EY, Holsinger C, DeMonte F, Kupferman M. Robotic endoscopic surgery of the skull base: a novel surgical approach. Arch Otolaryngol Head Neck Surg. 2007 Dec;133(12):1209-1214.

[6] Harvey RJ, Winder M, Parmar P, Lund V. Endoscopic skull base surgery for sinonasal malignancy. Otolaryngol Clin North Am. 2011 Oct;44(5):1081-1140.

doi: 10.1016/j.otc.2011.06.020. Available from: http://dx.doi.org/10.1016/j.otc.2011.06.020.
[7] Leong SC1, White PS. A comparison of aesthetic proportions between the Oriental and Caucasian nose. Clin Otolaryngol Allied Sci. 2004 Dec;29(6):672-6.

[8] O'Malley BW, Jr, Weinstein GS. Robotic skull base surgery: preclinical investigations to human clinical application. Arch Otolaryngol Head Neck Surg. 2007 Dec;133(12):1215-1219.

[9] Schwartz TH, Fraser JF, Brown S, Tabaee A, Kacker A, Anand VK. Endoscopic cranial base surgery: classification of operative approaches. Neurosurgery. 2008 May;62(5):991-1002. doi: 10.1227/01.neu.0000325861.06832.06.

Available

from: http://dx.doi.org/10.1227/01.neu.0000325861.0 6832.06.

[10] PCL - Point Cloud Library (PCL) http://pointclouds.org/

[11] Tombari, F., \& Stefano, L. Di. (2010). Object Recognition in 3D Scenes with Occlusions and Clutter by Hough Voting. In 2010 Fourth Pacific-Rim Symposium on Image and Video Technology (pp. 349-355). https://doi.org/10.1109/PSIVT.2010.65

[12] "Tratamiento de los tumores de hipófisis (PDQ $\left.{ }^{\circledR}\right)$ - Versión para pacientes - National Cancer Institute.” [Online]. Available: https://www.cancer.gov/espanol/tipos/hipofisis/ paciente/tratamiento-hipofisis-pdq. [Accessed: 18-Jun-2019]

[13] "Tumores de la Región Selar | NeuroWikia." [Online]. Available: http://www.neurowikia.es/content/tumores-dela-región-selar. [Accessed: 18-Jun-2019].

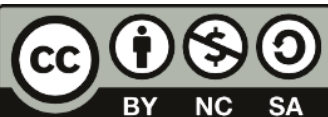
(C) 2019 by the authors. Submitted for possible open access publication under the terms and conditions of the Creative Commons Attribution CC BY-NC-SA 4.0 license (https://creativecommons.org/licenses/bync-sa/4.0/deed.es). 